\title{
Momentum Distribution Functions in a One-Dimensional Extended Periodic Anderson Model
}

\section{Hagymási, J. Sólyom, and Ö. Legeza}

Strongly Correlated Systems "Lendület" Research Group, Institute for Solid State Physics and Optics, MTA Wigner Research Centre for Physics, P.O. Box 49, Budapest 1525, Hungary

Correspondence should be addressed to I. Hagymási; hagymasi.imre@wigner.mta.hu

Received 7 May 2015; Accepted 31 August 2015

Academic Editor: Victor V. Moshchalkov

Copyright (C) 2015 I. Hagymási et al. This is an open access article distributed under the Creative Commons Attribution License, which permits unrestricted use, distribution, and reproduction in any medium, provided the original work is properly cited.

\begin{abstract}
We study the momentum distribution of the electrons in an extended periodic Anderson model, where the interaction, $U_{c f}$, between itinerant and localized electrons is taken into account. In the symmetric half-filled model, due to the increase of the interorbital interaction, the $f$ electrons become more and more delocalized, while the itinerancy of conduction electrons decreases. Above a certain value of $U_{c f}$ the $f$ electrons become again localized together with the conduction electrons. In the less than half-filled case, we observe that $U_{c f}$ causes strong correlations between the $f$ electrons in the mixed valence regime.
\end{abstract}

\section{Introduction}

Heavy-fermion and mixed valence systems are still active research fields in spite of the major achievements of the past few decades $[1,2]$. The discovery of a new critical point in the pressure-temperature phase diagram of $\mathrm{CeCu}_{2} \mathrm{Ge}_{2}$ and $\mathrm{CeCu}_{2} \mathrm{Si}_{2}$ has attracted much attention both experimentally [3-8] and theoretically [9-14]. It is believed that the appearance of the new critical point is due to the critical valence fluctuations of the Ce ion. The simplest model, which contains the essential physics of rare-earth compounds, is the periodic Anderson model:

$$
\begin{aligned}
\mathscr{H}_{\mathrm{PAM}}= & -t \sum_{\langle i j\rangle, \sigma} \widehat{c}_{\sigma \sigma}^{\dagger} \widehat{c}_{j \sigma}-V \sum_{j, \sigma}\left(\widehat{f}_{j \sigma}^{\dagger} \widehat{c}_{j \sigma}+\widehat{c}_{j \sigma}^{\dagger} \widehat{f}_{j \sigma}\right) \\
& +\varepsilon_{f} \sum_{j, \sigma} \widehat{n}_{j \sigma}^{f}+U_{f} \sum_{j} \widehat{n}_{j \uparrow}^{f} \widehat{n}_{j \downarrow}^{f},
\end{aligned}
$$

where the notation is standard and $W=4 t$ is taken as the energy unit. It is known, however, that the mixed valence regime appears always in this model as a smooth crossover, and valence fluctuations do not become critical for any choice of the parameters. A local Coulomb interaction between the conduction and localized electrons is needed for the appearance of a sharp transition and critical valence fluctuations [14]. Therefore we consider the following Hamiltonian:

$$
\mathscr{H}=\mathscr{H}_{\mathrm{PAM}}+U_{c f} \sum_{j} \widehat{n}_{j}^{f} \widehat{n}_{j}^{c}
$$

Previous studies revealed how $U_{c f}$ affects the mixed valence regime and it has been shown that a first-order valence transition and a quantum critical point may appear due to $U_{c f}$ $[11,15-17]$. The effect of $U_{c f}$ in the Kondo regime has also been addressed both in infinite $[18,19]$ and one spatial dimensions [20]. Namely, in infinite dimensions the symmetric model for small hybridization $(V \ll W)$ displays antiferromagnetic order for small $U_{c f}$ which, however, disappears for large $U_{c f}$ and charge order develops. In contrast, there is no such phase transition in one dimension due to the enhanced quantum fluctuations; however, for small and large $U_{c f}$ the spinspin and density-density correlation function, respectively, exhibits the slowest decay. Between these two regimes there is a narrow region, where the local singlet formation is significantly enhanced. It is worth noting that, besides $U_{c f}$, the role of the interaction between conduction electrons has also been studied [21, 22]; however, this does not lead to a sharp valence transition. 
Our goal in this paper is to investigate the momentum distribution of the electrons in one dimension. It is known that in higher dimensions they exhibit a jump at the Fermi momentum, whose size can be used to extract the energy dependence of the self-energies, from which the many-body enhancement factor of the effective mass can be obtained. Although in one dimension there is no such jump at the Fermi momentum, just a sharp change, they provide direct information about the spatial distribution of the electrons and the content of conduction and $f$-electron states in the quasiparticle bands, while the previous quantum information analysis [20] gave only an indirect description of these quantities. We address the question of how they are modified by switching on $U_{c f}$ both in the integer and mixed valence regimes. The density-matrix renormalizationgroup algorithm (DMRG) [23-27] is applied, which allows the accurate determination of ground state properties. We have used the dynamic block-state selection algorithm [28, 29 ] in which the threshold value of the quantum information loss, $\chi$, is set a priori. We have taken $\chi=10^{-5}$. A maximum of 2000 block states is needed to achieve this accuracy, and the largest truncation error was in the order of $10^{-6}$. We investigated chains up to a maximum length $L=80$ with open boundary conditions and performed 8-12 sweeps.

\section{Results at Half-Filling}

The nondegenerate version of the periodic Anderson model can hold up to $n_{\max }=4$ electrons per lattice site, the average number of $c$ and $f$ electrons per site, $n^{c}$ and $n^{f}$, respectively, can vary between zero and two. The filling will refer to the ratio of the total electron density per site $\left(n^{c}+n^{f}\right)$. In what follows we consider the symmetric half-filled model, where $n^{f}=1$, and calculate the momentum distribution of conduction and $f$ electrons which are defined as

$$
\begin{aligned}
& n^{c}(k)=\frac{1}{2} \sum_{\sigma}\left\langle c_{k \sigma}^{\dagger} c_{k \sigma}\right\rangle, \\
& n^{f}(k)=\frac{1}{2} \sum_{\sigma}\left\langle f_{k \sigma}^{\dagger} f_{k \sigma}\right\rangle .
\end{aligned}
$$

Our DMRG calculation was performed in real space; therefore these quantities can be obtained by Fourier transforming of the corresponding single particle density matrices; namely,

$$
\begin{aligned}
& n^{c}(k)=\frac{1}{2} \sum_{j l \sigma} e^{i k(j-l)}\left\langle c_{j \sigma}^{\dagger} c_{l \sigma}\right\rangle, \\
& n^{f}(k)=\frac{1}{2} \sum_{j l \sigma} e^{i k(j-l)}\left\langle f_{j \sigma}^{\dagger} f_{l \sigma}\right\rangle,
\end{aligned}
$$

where $k=2 \pi n / L$ and $n=-L / 2-1, \ldots, L / 2$. In our case these are symmetric functions; therefore we consider only the nonnegative $k$ values.

Before going into the details of the numerical results, we briefly recall the case when $U_{f}=U_{c f}=0$ which is easily

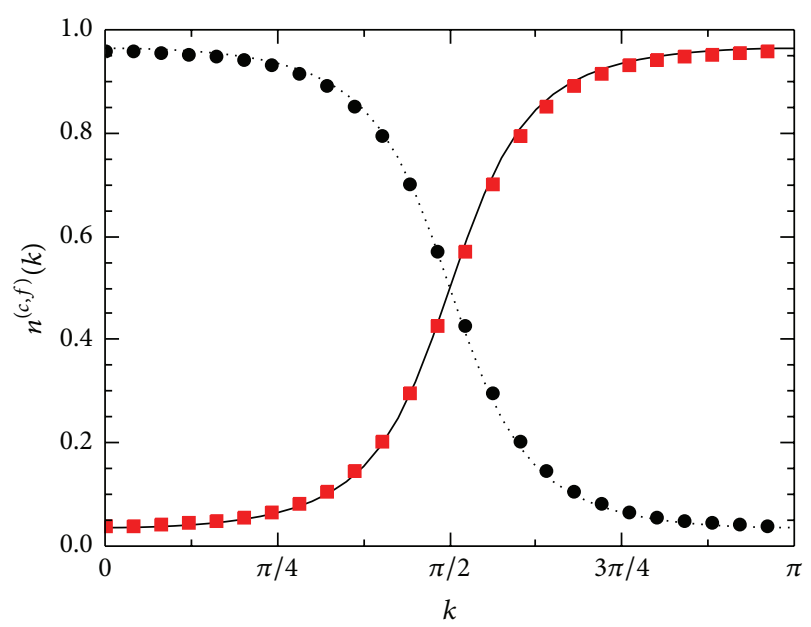

FIGURE 1: Momentum distribution functions of the conduction and $f$ electrons in the noninteracting case $\left(U_{f}=U_{c f}=0, V / W=0.1\right.$, $\varepsilon_{f}=0$ ). The solid and dotted lines are obtained from (6) and (7), respectively. The symbols (bullet and red square) denote the DMRG results for $L=50$.

solvable. Thereby the Hamiltonian can be diagonalized by an unitary transformation

$$
\begin{aligned}
& \alpha_{k}^{(-)}=-v_{k} c_{k}+u_{k} f_{k}, \\
& \alpha_{k}^{(+)}=u_{k} c_{k}+v_{k} f_{k},
\end{aligned}
$$

where $\alpha_{k}^{(-)}\left(\alpha_{k}^{(+)}\right)$creates a quasiparticle in the lower (upper) hybridized band with mixing amplitudes:

$$
\begin{aligned}
& u_{k}^{2}=\frac{1}{2}\left[1-\frac{\varepsilon_{k}-\varepsilon_{f}}{\sqrt{\left(\varepsilon_{k}-\varepsilon_{f}\right)^{2}+4 V^{2}}}\right], \\
& v_{k}^{2}=\frac{1}{2}\left[1+\frac{\varepsilon_{k}-\varepsilon_{f}}{\sqrt{\left(\varepsilon_{k}-\varepsilon_{f}\right)^{2}+4 V^{2}}}\right] ;
\end{aligned}
$$

in our case $\varepsilon_{k}=-2 t \cos k$ and the Fermi momentum is at the boundary of the Brillouin zone since the lower band is completely filled. It is easily seen that the momentum distribution functions provide information about the mixing amplitudes, namely, the portion of conduction and $f$ states in the hybridized band:

$$
\begin{aligned}
& n^{c}(k)=v_{k}^{2} \\
& n^{f}(k)=u_{k}^{2}
\end{aligned}
$$

The momentum distribution in the noninteracting system is shown in Figure 1 compared with the DMRG results. The small discrepancy between the two results is attributed to the open boundary condition used in DMRG. In the following we investigate how the interactions modify the above results, using again the DMRG method with open 


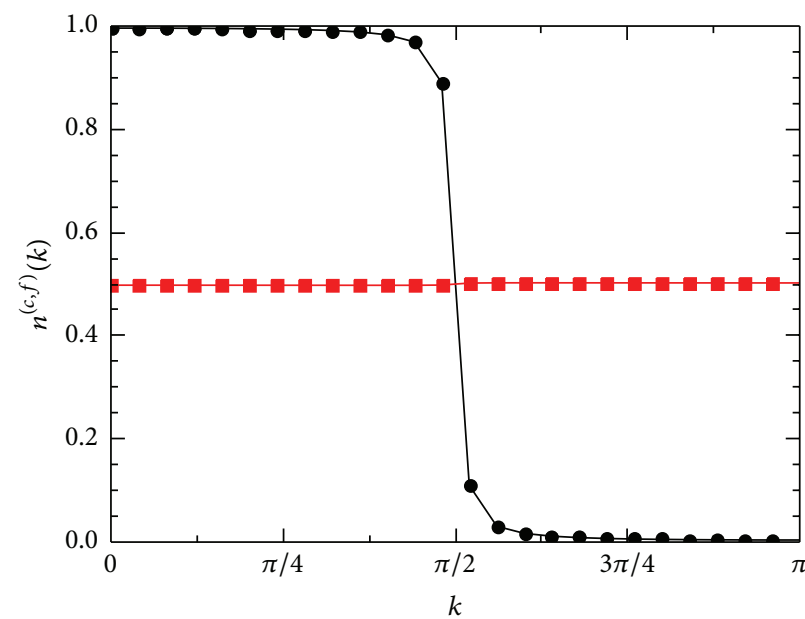

(a)

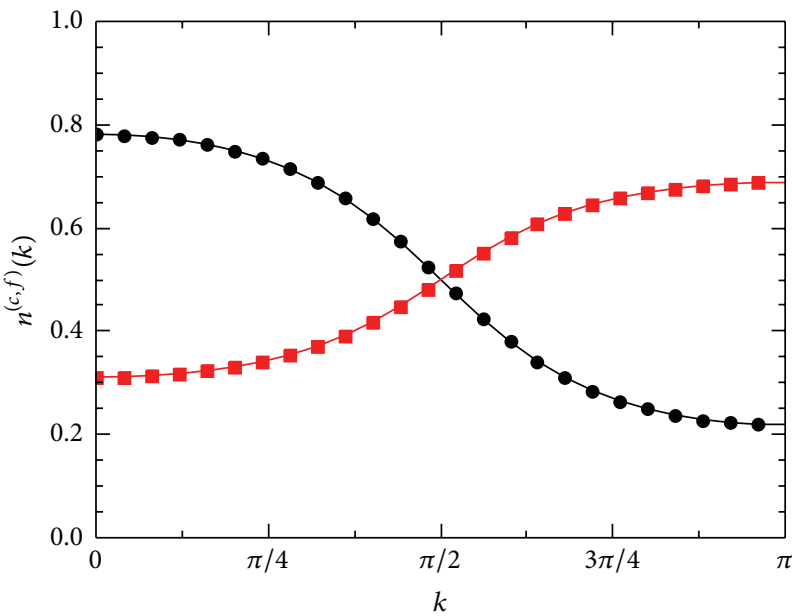

(c)

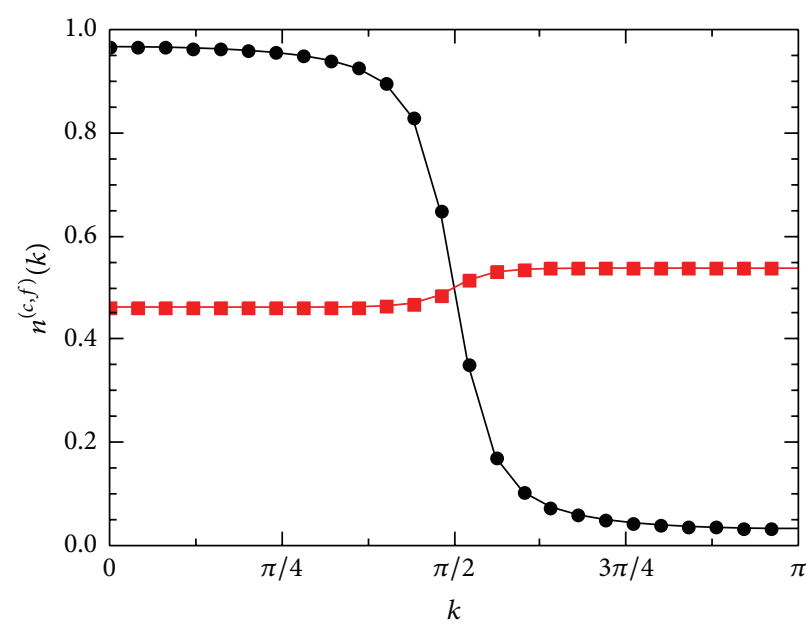

(b)

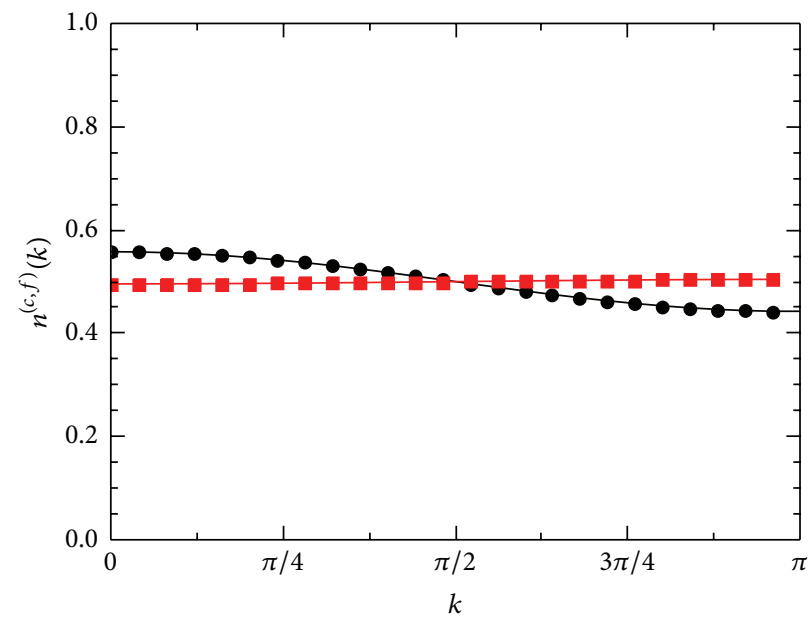

(d)

Figure 2: Momentum distribution functions of the conduction (bullet) and $f$ (red square) electrons for $L=50$ and $n=2$. Panels (a), (b), (c), and (d) correspond to $U_{c f} / W=0,1.5,1.7$, and 3; furthermore $U_{f} / W=3, V / W=0.1$, and $\varepsilon_{f}=-U_{f} / 2$ in all cases. The lines are guides to the eye.

boundary condition. We checked for short systems that the momentum distributions of the interacting system calculated with periodic and open boundary conditions are in good agreement within our error margin.

It has been pointed out $[20,30]$ that strong $U_{f}$ leads to localization of the $f$ electrons, since the number of doubly occupied $f$ levels is negligible and the ground state is a collective singlet. This is what we see in Figure 2(a); namely, $n^{f}(k)$ hardly depends on $k$ in the Kondo regime, while the distribution of conduction electrons is just slightly affected by $U_{f}$. The quantum information analysis [20] showed that as $U_{c f}$ is switched on more and more doubly occupied $f$ sites are created, therefore the $f$ electrons become less localized in real space. Finally, when $U_{c f}$ is large the $c$ and $f$ electrons tend to avoid each other and the sites are occupied by two $c$ or two $f$ electrons in an alternating fashion. Now we examine how these features are reflected in the momentum distributions. As $U_{c f}$ is switched on, the wave number dependence of the conduction electrons becomes weaker and weaker and therefore less itinerant in real space, while the distribution of the $f$ electrons becomes more and more dispersive as it can be seen in Figures 2(b) and 2(c). Above $U_{c f} \approx U_{f} / 2+$ $W / 4$ both distributions hardly depend on the wave number as it is observed in Figure 2(d). That is, the behavior of the momentum distributions agrees well with the results of the entropy analysis.

\section{Away from Half-Filling}

In the previous section we considered the half-filled case. Now we discuss what happens when the ground state is metallic and fix the electron density at $n=1.75$. It has been shown $[15,16]$ that a stable mixed valence regime appears around $n^{f}=2-n$ in the presence of $U_{c f}$, and strong enough $U_{c f}$ leads to a first-order transition between the Kondo and mixed valence states as $\varepsilon_{f}$ is varied. One can observe, in Figure 3, that for strong $U_{c f}$ a stable mixed valence regime appears indeed, and the change of the valence becomes 


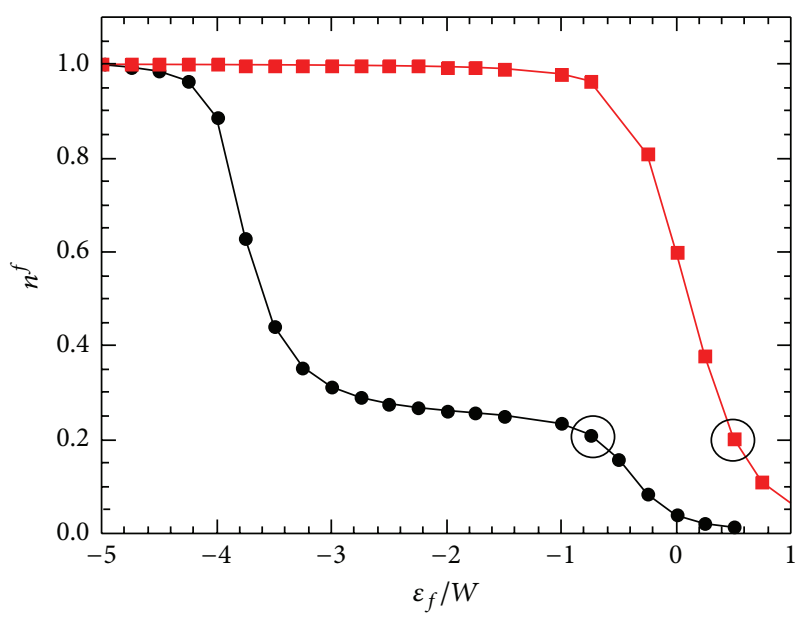

FIgURE 3: The $f$-level occupancy as a function of $\varepsilon_{f}$ for $L=80, U_{c f}=$ 0 (red square) and $U_{c f} / W=4$ (bullet); furthermore $U_{f} / W=10$ and $V / W=0.2$. The circled data points are used in the comparison in Figure 4 . The lines are guides to the eye.

sharp. In the following we investigate how the momentum distribution of the electrons changes due to $U_{c f}$ in the mixed valence regime. This is shown in Figure 4 , where $\varepsilon_{f}$, indicated by the circles around the data points in Figure 3, was chosen such that the occupancy of the $f$ level is nearly the same in the two cases. One can clearly see that both $n^{c}(k)$ and $n^{f}(k)$ change drastically around the Fermi momentum when $U_{c f}=0$. This is not surprising since our system is a Luttinger liquid, where a logarithmic singularity is expected to occur at the Fermi momentum. For a finite $U_{c f}$ a significant amount of the conduction and $f$ electrons is scattered above the Fermi momentum and the $k$-dependence of the electron densities is significantly reduced in both cases, which indicates a strongly correlated mixed valence state. It is worth noting that the distribution functions do not tend to zero above the Fermi momentum for strong $U_{c f}$. These results agree well with what has been obtained in infinite dimensions using the Gutzwiller wave function [16]; the main difference is the absence of the discontinuity at the Fermi momentum due to the onedimensional property of the model.

\section{Conclusions}

We have investigated an extended periodic Anderson model with an additional Coulomb interaction using the DMRG algorithm to better understand its effect on the momentum distribution of the electrons. In the half-filled, symmetric model (in the Kondo regime), switching on $U_{c f}$ results in the increased itinerancy of the $f$ electrons; however, above a certain value of $U_{c f}$ it tends to localize $f$ electrons again. The itinerancy of the conduction electrons is gradually reduced as $U_{c f}$ is increased. These results agree well with what has been obtained by quantum information analysis [20]. We also investigated what happens when the system is less than half-filled; that is, the ground state is metallic. It has been revealed that in the mixed valence regime $U_{c f}$ makes

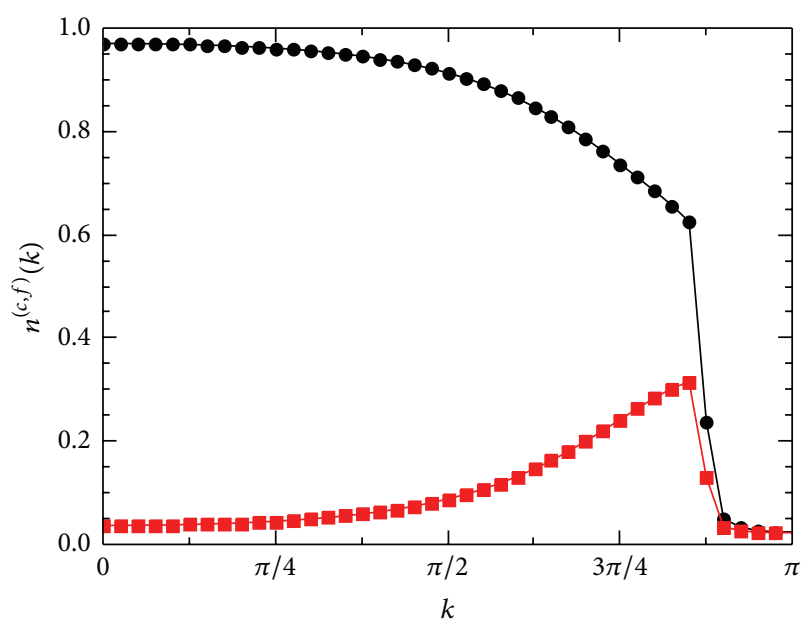

(a)

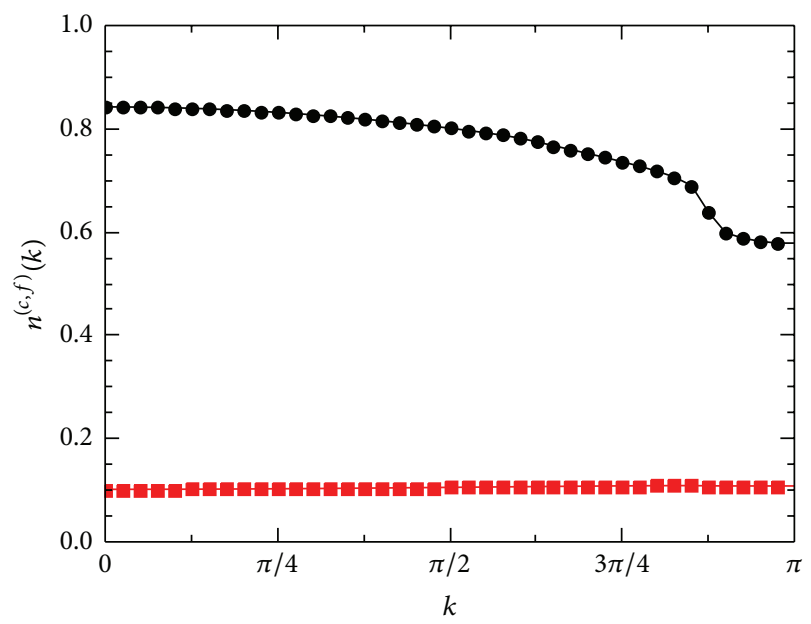

(b)

FIgURE 4: Momentum distribution functions of the conduction (bullet) and $f$ (red square) electrons for $L=80$ and $n=1.75$. Panels (a) and (b) correspond to $U_{c f} / W=0, \varepsilon_{f} / W=0.5$ and $U_{c f} / W=4, \varepsilon_{f} / W=-0.75$, respectively; furthermore $U_{f} / W=10$ and $V / W=0.2$ in all cases. The lines are guides to the eye.

both the conduction and $f$ electrons more correlated. These findings agree qualitatively well with the properties of the infinite dimensional model, although, as expected for a onedimensional model, there is no sharp Fermi edge. This could be analyzed further using the momentum space version of the DMRG method [31-34].

\section{Conflict of Interests}

The authors declare that there is no conflict of interests regarding the publication of this paper.

\section{Acknowledgments}

This work was supported in part by the Hungarian Research Fund (OTKA) through Grants nos. K 100908 and NN110360. 


\section{References}

[1] A. C. Hewson, The Kondo Problem to Heavy Fermions, Cambridge University Press, Cambridge, UK, 1993.

[2] P. Fazekas, Lecture Notes on Electron Correlation and Magnetism, World Scientific, Singapore, 1999.

[3] H. Q. Yuan, F. M. Grosche, M. Deppe, C. Geibel, G. Sparn, and F. Steglich, "Observation of two distinct superconducting phases in $\mathrm{CeCu}_{2} \mathrm{Si}_{2}$," Science, vol. 302, no. 5653, pp. 2104-2107, 2003.

[4] A. T. Holmes, D. Jaccard, and K. Miyake, "Signatures of valence fluctuations in $\mathrm{CeCu}_{2} \mathrm{Si}_{2}$ under high pressure," Physical Review $B$, vol. 69, no. 2, Article ID 024508, 11 pages, 2004.

[5] H. Q. Yuan, F. M. Grosche, M. Deppe, G. Sparn, C. Geibel, and F. Steglich, "Non-Fermi liquid states in the pressurized $\mathrm{CeCu}_{2}\left(\mathrm{Si}_{1-\mathrm{x}} \mathrm{Ge}_{\mathrm{x}}\right)_{2}$ system: two critical points," Physical Review Letters, vol. 96, no. 4, Article ID 047008, 2006.

[6] K. Fujiwara, Y. Hata, K. Kobayashi et al., "High pressure NQR measurement in $\mathrm{CeCu}_{2} \mathrm{Si}_{2}$ up to sudden disappearance of superconductivity," Journal of the Physical Society of Japan, vol. 77, no. 12, Article ID 123711, 2008.

[7] E. Lengyel, M. Nicklas, H. S. Jeevan et al., "Pressure-induced change of the pairing symmetry in superconducting $\mathrm{CeCu}_{2} \mathrm{Si}_{2}$," Physical Review B, vol. 80, no. 14, Article ID 140513, 4 pages, 2009.

[8] J.-P. Rueff, S. Raymond, M. Taguchi et al., "Pressure-induced valence crossover in superconducting $\mathrm{CeCu}_{2} \mathrm{Si}_{2}$," Physical Review Letters, vol. 106, no. 18, Article ID 186405, 2011.

[9] Y. Onishi and K. Miyake, "Enhanced valence fluctuations caused by f-c coulomb interaction in Ce-based heavy electrons: possible origin of pressure-induced enhancement of superconducting transition temperature in $\mathrm{CeCu}_{2} \mathrm{Ge}_{2}$ and related compounds," Journal of the Physical Society of Japan, vol. 69, no. 12, pp. 3955-3964, 2000.

[10] K. Miyake and H. Maebashi, "Huge enhancement of impurity scattering due to critical valence fluctuations in a Ce-based heavy electron system," Journal of the Physical Society of Japan, vol. 71, no. 4, pp. 1007-1010, 2002.

[11] S. Watanabe, M. Imada, and K. Miyake, "Superconductivity emerging near quantum critical point of valence transition," Journal of the Physical Society of Japan, vol. 75, no. 4, Article ID 043710, 2006.

[12] S. Watanabe, M. Imada, and K. Miyake, "Quantum valence criticality and superconductivity," Journal of Magnetism and Magnetic Materials, vol. 310, no. 2, part 1, pp. 841-843, 2007.

[13] A. T. Holmes, D. Jaccard, and K. Miyake, "Valence instability and superconductivity in heavy fermion systems," Journal of the Physical Society of Japan, vol. 76, no. 5, Article ID 051002, 2007.

[14] K. Miyake, "New trend of superconductivity in strongly correlated electron systems," Journal of Physics: Condensed Matter, vol. 19, no. 12, Article ID 125201, 2007.

[15] Y. Saiga, T. Sugibayashi, and D. S. Hirashima, "Valence instability and the quantum critical point in an extended periodic anderson model: analysis based on the dynamical mean field theory," Journal of the Physical Society of Japan, vol. 77, no. 11, Article ID 114710, 2008.

[16] K. Kubo, "Gutzwiller method for an extended periodic anderson model with the $c-f$ coulomb interaction," Journal of the Physical Society of Japan, vol. 80, no. 11, Article ID 114711, 8 pages, 2011.

[17] I. Hagymási, K. Itai, and J. Sólyom, "Quantum criticality and first-order transitions in the extended periodic Anderson model," Physical Review B, vol. 87, no. 12, Article ID 125146, 2013.
[18] T. Yoshida, T. Ohashi, and N. Kawakami, "Effects of conduction electron correlation on heavy-fermion systems," Journal of the Physical Society of Japan, vol. 80, no. 6, Article ID 064710, 2011.

[19] T. Yoshida and N. Kawakami, "Interorbital correlation effects on heavy-electron systems," Physical Review B, vol. 85, Article ID 235148, 2012.

[20] I. Hagymási, J. Sólyom, and Ö. Legeza, "Interorbital interaction in the one-dimensional periodic Anderson model: density matrix renormalization group study," Physical Review B, vol. 90, no. 12, Article ID 125137, 2014.

[21] S. Ramasesha and K. Das, "Valence-bond theory of the Kondo lattice model in one dimension," Physical Review B, vol. 42, no. 16, Article ID 10682, 1990.

[22] I. Hagymási, K. Itai, and J. Sólyom, “Periodic Anderson model with correlated conduction electrons: variational and exact diagonalization study," Physical Review B, vol. 85, no. 23, Article ID 235116, 2012.

[23] S. R. White, "Density matrix formulation for quantum renormalization groups," Physical Review Letters, vol. 69, no. 19, pp. 2863-2866, 1992.

[24] S. R. White, "Density-matrix algorithms for quantum renormalization groups," Physical Review B, vol. 48, no. 14, pp. 1034510356, 1993.

[25] U. Schollwöck, "The density-matrix renormalization group," Reviews of Modern Physics, vol. 77, no. 1, pp. 259-315, 2005.

[26] R. M. Noack and S. R. Manmana, "Diagonalization- and numerical renormalization-group-based methods for interacting quantum systems," AIP Conference Proceedings, vol. 789, pp. 93$163,2005$.

[27] K. A. Hallberg, "New trends in density matrix renormalization," Advances in Physics, vol. 55, no. 5-6, pp. 477-526, 2006.

[28] Ö. Legeza, J. Röder, and B. A. Hess, "Controlling the accuracy of the density-matrix renormalization-group method: the dynamical block state selection approach," Physical Review B, vol. 67, no. 12, Article ID 125114, 2003.

[29] Ö. Legeza and J. Sólyom, "Quantum data compression, quantum information generation, and the density-matrix renormalization-group method," Physical Review B, vol. 70, no. 20, Article ID 205118, 2004.

[30] H. Shiba, "Properties of strongly correlated Fermi liquid in valence fluctuation system-a variational Monte-Carlo study," Journal of the Physical Society of Japan, vol. 55, no. 8, pp. 2765$2776,1986$.

[31] T. Xiang, "Density-matrix renormalization-group method in momentum space," Physical Review B, vol. 53, no. 16, pp. R10445-R10448, 1996.

[32] S. Nishimoto, E. Jeckelmann, F. Gebhard, and R. M. Noack, "Application of the density matrix renormalization group in momentum space," Physical Review B, vol. 65, no. 16, Article ID 165114, 2002.

[33] Ö. Legeza and J. Sólyom, "Optimizing the density-matrix renormalization group method using quantum information entropy," Physical Review B, vol. 68, no. 19, Article ID 195116, 19 pages, 2003.

[34] G. Ehlers, J. Sólyom, Ö. Legeza, and R. M. Noack, "Entanglement structure of the Hubbard model in momentum space," http://arxiv.org/abs/1508.07477. 

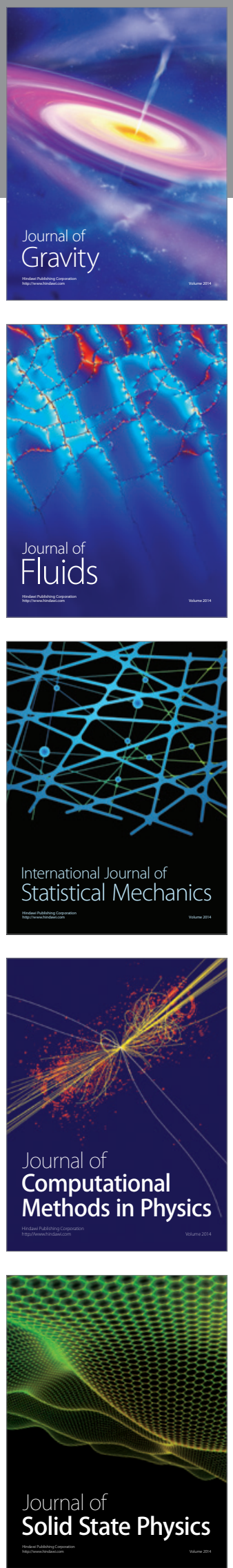

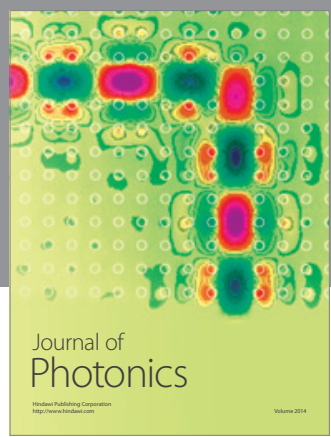

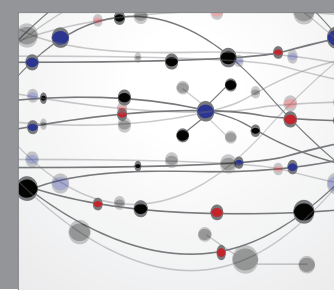

The Scientific World Journal

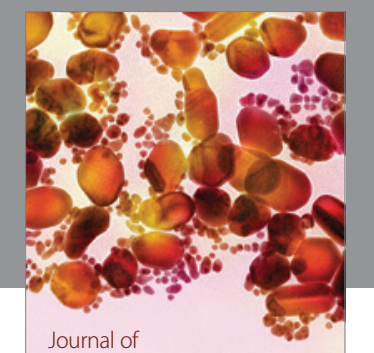

Soft Matter
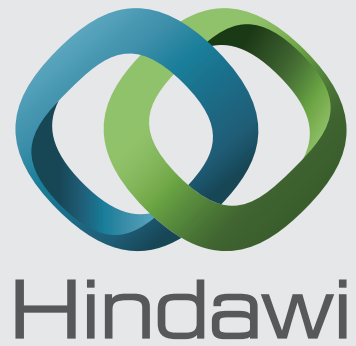

Submit your manuscripts at

http://www.hindawi.com
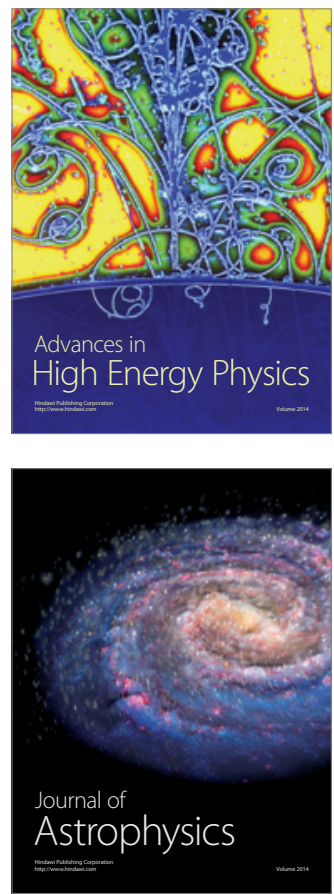
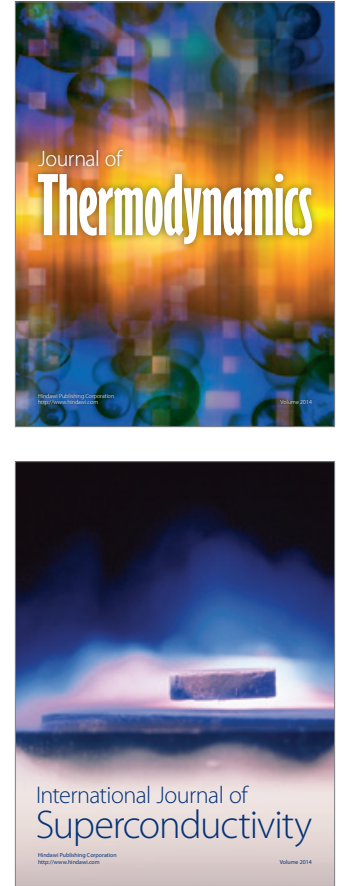
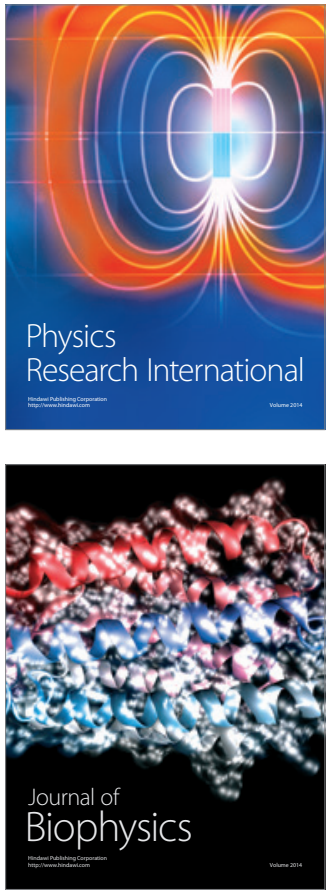
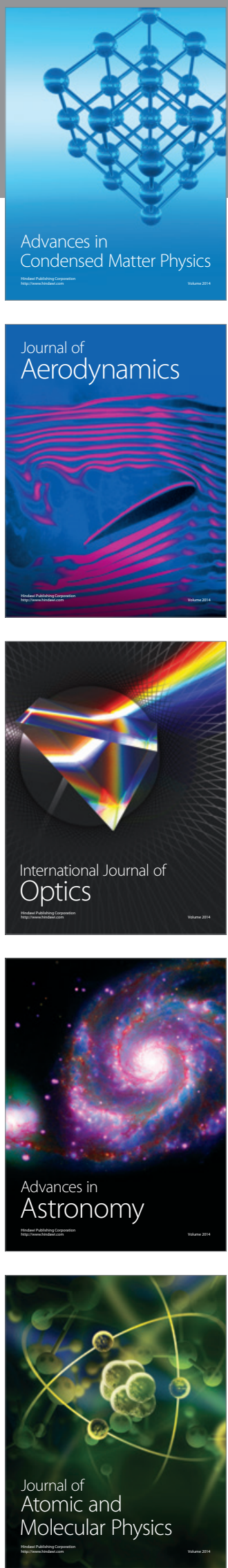